\section{TATRA \\ MOUNTaiNS \\ Mathematical Publications}

DOI: $10.2478 / \mathrm{tmmp}-2013-0020$

Tatra Mt. Math. Publ. 55 (2013), 67-75

\title{
ABSTRACT PYTHAGOREAN THEOREM AND CORRESPONDING FUNCTIONAL EQUATIONS
}

\author{
Roman Ger
}

\begin{abstract}
Motivated by a result of [Lucio R. Berrone: The associativity of the Pythagorean law, Amer. Math. Monthly 116 (2009), 936-939] we deal with the functional equation

$$
f(x+y)=f(x)+f(y)+2 f(\Phi(x, y))
$$

and its variants to distinguish the classical Pythagorean theorem from among its numerous abstract generalizations.
\end{abstract}

\section{Motivation}

Let $A B C$ be a right triangle based on its hypothenuse $A B$. Denote by $H$ the spot of the height corresponding to the hypothenuse and let $x, y$ and $\Phi(x, y)$ stand for the lengths of the segments $A H, H B$ and $C H$, respectively. The classical "school-formulas" based on the Pythagorean Theorem give then the following equality

$$
f(x+y)=f(x)+f(y)+2 f(\Phi(x, y))
$$

with

$$
f(x)=x^{2} \quad \text { and } \quad \Phi(x, y)=\sqrt{x y}, \quad x, y \in(0, \infty) .
$$

$\mathrm{Luci}$ o R. B e r r o n e [1] has requested the reader to forget his or her knowledge of the Pythagorean proposition and to assume that equation (E) is satisfied provided that

- $f:(0, \infty) \longrightarrow(0, \infty)$ is continuous and strictly monotonic;

- $\Phi:(0, \infty) \times(0, \infty) \longrightarrow(0, \infty)$ is reflexive, i.e., $\Phi(x, x)=x, x \in(0, \infty)$.

Lucio R. Berrone's fundamental assumption states that the associative operation

$$
F(x, y):=f^{-1}(f(x)+f(y)), \quad(x, y) \in(0, \infty) \times(0, \infty),
$$

(C) 2013 Mathematical Institute, Slovak Academy of Sciences.

2010 Mathematics Subject Classification: 39B22, 51M05.

Keywords: Pythagorean Law, height function, associativity, quasi-arithmetic mean. 
is homogeneous, i.e., $F(t x, t y)=t F(x, y)$ for all $x, y, t \in(0, \infty)$. Under these assumptions he proves that

$$
F(x, y)=\sqrt{x^{2}+y^{2}} \text { and } \Phi(x, y)=\sqrt{x \cdot y},
$$

which forces $f$ to be of the form $f(x)=c x^{2}, x \in(0, \infty)$, with some nonzero constant $c$.

It seems that Berrone's problem is ill posed for two (independent) reasons:

- it is tacitly assumed that if the triangle $\mathrm{AHC}$ is a right one, then so is the triangle $\mathrm{BHC}$;

- the homogeneity assumption for $F$ is hanging in the air (or rather says implicitely that we deal with the affine geometry).

The tacitly adopted assumption was formalized in 1982 by L. Dubikajt is [2] in his S-geometry axiomatics. With the aid of functional equation methods I have proved in [3] that any theorem derived in S-geometry of L. Dubikajtis yields a common property of the classical Euclidean, hyperbolic and elliptic geometry; on the other hand, these axioms admit no essentially different geometries. The corresponding orthogonality functions are:

- $f(x)=a x^{2}, x \in(0, \infty)$,

- $f(x)=a \ln \cosh b x, x \in(0, \infty)$,

- $f(x)=-a \ln \cos b x, x \in(0, m), m \in(0, \infty), 2 m|b| \leq \pi$,

respectively; here $a>0$ and $b \neq 0$ are arbitrary constants.

The corresponding abstract Pythagorean theorems are:

- $F(x, y)=\sqrt{x^{2}+y^{2}},(x, y) \in(0, \infty) \times(0, \infty)$,

- $F(x, y)=\operatorname{arc} \cosh (\cosh x \cdot \cosh y),(x, y) \in(0, \infty) \times(0, \infty)$,

- $F(x, y)=\arccos (\cos x \cdot \cos y),(x, y) \in(0, m) \times(0, m)$.

All three of them are associative and only the first one happens to be homogeneous.

\section{The simplest case}

To be precise, the effect presented at the end of the previous section has been achieved under the assumption that $f$ admits an extension onto $[0, \infty)$ such that

$$
f(0)=f^{\prime}(0)=0 \quad \text { and } \quad f^{\prime \prime}(0) \text { does exist. }
$$

If that is the case, with a surprisingly simple proof, we have also the following result. 
TheOrem 1. The only nonzero solutions $f:(0, \infty) \longrightarrow \mathbb{R}$, admitting a finite limit of the quotient $\frac{f(x)}{x^{2}}$ at zero, of the equation

$$
f(x+y)=f(x)+f(y)+2 f(\Phi(x, y))
$$

with a reflexive function $\Phi:(0, \infty) \times(0, \infty) \longrightarrow(0, \infty)$ are of the form

$$
f(x)=c \cdot x^{2} \quad \text { and } \quad \Phi(x, y)=\sqrt{x \cdot y}, \quad(x, y) \in(0, \infty) \times(0, \infty) .
$$

Proof. Put $y=x$ in (E) and apply the reflexivity of $\Phi$ to get the equality $f(2 x)=4 f(x)$ for all $x \in(0, \infty)$. Setting

$$
g(x)=\frac{f(x)}{x}, \quad x \in(0, \infty),
$$

we have

$$
\lim _{x \rightarrow 0^{+}} \frac{g(x)}{x}=: c \in \mathbb{R} \quad \text { as well as } \quad g(2 x)=2 g(x), x \in(0, \infty) .
$$

By a simple induction, for every positive integer $n$, we also obtain the equalities

$$
g\left(\frac{1}{2^{n}} x\right)=\frac{1}{2^{n}} g(x), \quad x \in(0, \infty),
$$

whence, finally,

$$
\frac{g(x)}{x}=\frac{\frac{1}{2^{n}} g(x)}{\frac{1}{2^{n}} x}=\frac{g\left(\frac{1}{2^{n}} x\right)}{\frac{1}{2^{n}} x} \longrightarrow c \quad \text { as } \quad n \longrightarrow \infty .
$$

Consequently, for every $x \in(0, \infty)$, we get

$$
f(x)=g(x) x=c x^{2},
$$

as claimed, because clearly $c$ cannot vanish since, otherwise, we would have $f=0$. Jointly with (E) this formula gives also the desired form of $\Phi$ and finishes the proof.

\section{Differentiable solutions}

What about the situation where we have no knowledge whether or not a suitable extension of $f$ onto the closure of its domain exists? It seems reasonable that resigning from a slightly artificial homogeneity assumption upon the Pythagorean theorem

$$
F(x, y)=f^{-1}(f(x)+f(y)), \quad(x, y) \in(0, \infty) \times(0, \infty),
$$


we shall require the height function $\Phi$ to be a mean and, to keep some balance, to assume that this mean is quasi-arithmetic, i.e.,

$$
\Phi(x, y)=\varphi^{-1}\left(\frac{\varphi(x)+\varphi(y)}{2}\right), \quad(x, y) \in(0, \infty) \times(0, \infty),
$$

where $\varphi$ stands for a strictly monotonic and continuous real function on $(0, \infty)$.

Our first result in that direction reads as follows:

Theorem 2. Let $f, \varphi:(0, \infty) \longrightarrow \mathbb{R}$ be twice differentiable functions with nowhere vanishing derivatives $f^{\prime}$ and $\varphi^{\prime}$. Then the pair $(f, \varphi)$ yields a solution to the functional equation

$$
f(x+y)=f(x)+f(y)+2 f\left(\varphi^{-1}\left(\frac{\varphi(x)+\varphi(y)}{2}\right)\right), \quad x, y \in(0, \infty),
$$

if and only if there exist nonzero real constants $c$ and $a$, and a real constant $b$ such that

$$
f(x)=c \cdot x^{2} \quad \text { and } \quad \varphi(x)=a \log x+b, \quad x \in(0, \infty) .
$$

Proof. Put

$$
\Phi(x, y):=\varphi^{-1}\left(\frac{\varphi(x)+\varphi(y)}{2}\right), \quad x, y \in(0, \infty) .
$$

Then we have

$$
\varphi(\Phi(x, y))=\frac{\varphi(x)+\varphi(y)}{2}, \quad x, y \in(0, \infty),
$$

whence, for all $x, y \in(0, \infty)$ one has

$$
\varphi^{\prime}(\Phi(x, y)) \frac{\partial}{\partial x} \Phi(x, y)=\frac{1}{2} \varphi^{\prime}(x), \quad \text { and } \quad \varphi^{\prime}(\Phi(x, y)) \frac{\partial}{\partial y} \Phi(x, y)=\frac{1}{2} \varphi^{\prime}(y) .
$$

On the other hand,

and

$$
f^{\prime}(x+y)-f^{\prime}(x)=2 f^{\prime}(\Phi(x, y)) \frac{\partial}{\partial x} \Phi(x, y)
$$

$$
f^{\prime}(x+y)-f^{\prime}(y)=2 f^{\prime}(\Phi(x, y)) \frac{\partial}{\partial y} \Phi(x, y)
$$

for all $x, y \in(0, \infty)$, which jointly with the latter two relationships implies the equality

as well as

$$
\left[f^{\prime}(x+y)-f^{\prime}(x)\right] \varphi^{\prime}(\Phi(x, y))=f^{\prime}(\Phi(x, y)) \varphi^{\prime}(x)
$$

$$
\left[f^{\prime}(x+y)-f^{\prime}(y)\right] \varphi^{\prime}(\Phi(x, y))=f^{\prime}(\Phi(x, y)) \varphi^{\prime}(y) \neq 0,
$$


valid for every $x, y \in(0, \infty)$. Dividing these two equalities side by side, we arrive at

$$
\frac{f^{\prime}(x+y)-f^{\prime}(x)}{f^{\prime}(x+y)-f^{\prime}(y)}=\frac{\varphi^{\prime}(x)}{\varphi^{\prime}(y)}, \quad x, y \in(0, \infty) .
$$

This in turn, after obvious rearrangements, leads to

$$
\begin{aligned}
f^{\prime}(x+y) \frac{\varphi^{\prime}(y)-\varphi^{\prime}(x)}{y-x}= & f^{\prime}(x) \frac{\varphi^{\prime}(y)-\varphi^{\prime}(x)}{y-x} \\
& -\varphi^{\prime}(x) \frac{f^{\prime}(y)-f^{\prime}(x)}{y-x}, \quad x, y \in(0, \infty), x \neq y .
\end{aligned}
$$

Passing here to the limit as $y$ tends to $x$ we infer that

$$
f^{\prime}(2 x) \varphi^{\prime \prime}(x)=f^{\prime}(x) \varphi^{\prime \prime}(x)-\varphi^{\prime}(x) f^{\prime \prime}(x), \quad x \in(0, \infty) .
$$

Since on setting $y=x$ in $\left(\mathrm{E}_{\varphi}\right)$ we deduce that $f(2 x)=4 f(x)$ and, a fortiori, $f^{\prime}(2 x)=2 f^{\prime}(x), x \in(0, \infty)$, we obtain finally

$$
f^{\prime}(x) \varphi^{\prime \prime}(x)+\varphi^{\prime}(x) f^{\prime \prime}(x)=0, \quad x \in(0, \infty),
$$

i.e.,

$$
\left(f^{\prime}(x) \varphi^{\prime}(x)\right)^{\prime} \equiv 0 \quad \text { on }(0, \infty)
$$

which implies that

$$
f^{\prime}(x) \varphi^{\prime}(x) \equiv \text { const }=: c_{0} \neq 0 \quad \text { on }(0, \infty) .
$$

Going back to $(*)$ we get then

$$
\frac{f^{\prime}(x+y)-f^{\prime}(x)}{f^{\prime}(x+y)-f^{\prime}(y)}=\frac{f^{\prime}(y)}{f^{\prime}(x)}, \quad x, y \in(0, \infty) .
$$

Putting here $y=2 x$ and recalling that $f^{\prime}(2 x)=2 f^{\prime}(x)$, we obtain the system

$$
f^{\prime}(2 x)=2 f^{\prime}(x) \quad f^{\prime}(3 x)=3 f^{\prime}(x), \quad x \in(0, \infty),
$$

which gives

$$
f^{\prime}\left(2^{n} 3^{m} x\right)=2^{n} 3^{m} f^{\prime}(x), \quad x \in(0, \infty),
$$

for all integers $n, m$. It is well-known that the set $\left\{2^{n} 3^{m}: n, m\right.$ are integers $\}$ is dense in $(0, \infty)$ whence, due to the continuity of the map $f^{\prime}$, we derive its linearity:

$$
f^{\prime}(\lambda)=f^{\prime}(1) \lambda, \quad \lambda \in(0, \infty) .
$$

Therefore, there exist real numbers $c, d, c \neq 0$, such that

$$
f(x)=c x^{2}+d, \quad x \in(0, \infty) .
$$

Note that we have to have $d=0$ because of the equality $f(2 x)=4 f(x)$ valid for all positive $x$.

Going back to $(* *)$ we get then

$$
\varphi^{\prime}(x)=\frac{a}{x}, \quad x \in(0, \infty) \text { with } \quad a:=\frac{c_{0}}{2 c},
$$


which states that there exists a real constant $b$ such that

$$
\varphi(x)=a \log x+b, \quad x \in(0, \infty)
$$

and completes the proof.

Remark. Plainly, the function $\varphi(x)=a \log x+d, x \in(0, \infty)$, yields a generator for the geometric mean:

$$
\Phi(x, y)=\varphi^{-1}\left(\frac{\varphi(x)+\varphi(y)}{2}\right)=\sqrt{x y}, \quad x, y \in(0, \infty) .
$$

Any other generator $\psi$ is of the same form because having the equality

$$
\psi^{-1}\left(\frac{\psi(x)+\psi(y)}{2}\right)=\varphi^{-1}\left(\frac{\varphi(x)+\varphi(y)}{2}\right)
$$

valid for all positive $x$ and $y$, we infer that $\psi \circ \varphi^{-1}$ is affine and monotonic whence $\psi\left(\varphi^{-1}(x)\right)=\lambda x+\mu, x \in(0, \infty)$, with some real constants $\lambda, \mu, \lambda \neq 0$, yielding the equality $\psi=\lambda \varphi+\mu$.

\section{Optimal setting of the problem}

The associative operation

$$
(0, \infty) \times(0, \infty) \ni(x, y) \longmapsto \sqrt{x^{2}+y^{2}}
$$

and the geometric mean

$$
(0, \infty) \times(0, \infty) \ni(x, y) \longmapsto \sqrt{x y}
$$

are of the form

$$
F(x, y)=f^{-1}(f(x)+f(y)) \quad \text { and } \quad \Phi(x, y)=\varphi^{-1}\left(\frac{\varphi(x)+\varphi(y)}{2}\right),
$$

respectively, where the generators $f$ and $\varphi$ are assumed to be strictly monotonic. In the present section we shall study equation (E) with the only additional assumption on $\varphi$ to be continuous. The basic proof tool will be the following (JMP)-theorem:

Theorem (A. Jár a i, G y. M a ks a and Z s. Páles [4]). Let $h:(0, \infty) \longrightarrow \mathbb{R}$ be a strictly monotonic function and let

$$
D:=\{h(x)+h(y): x, y \in(0, \infty)\} .
$$

Assume that a function $g: D \longrightarrow \mathbb{R}$ is strictly monotonic. If a function $f:(0, \infty) \longrightarrow \mathbb{R}$ is a solution to the functional equation

$$
f(x+y)=f(x)+f(y)+g(h(x)+h(y)),
$$


then there exist an additive function $A: \mathbb{R} \longrightarrow \mathbb{R}$ and four real constants $\alpha, \beta, \gamma, \delta$ such that $\alpha \beta \neq 0$ and $f$ has one of the following forms:

(i) $f(x)=\alpha \log (\cosh (\beta x+\gamma))+A(x)+\delta$;

(ii) $f(x)=\alpha \log (\sinh (\beta x+\gamma))+A(x)+\delta \quad$ (here $\beta>0$ and $\gamma \geq 0)$;

(iii) $f(x)=\alpha e^{\beta x}+A(x)+\delta$;

(iv) $f(x)=\alpha \log (x+\gamma)+A(x)+\delta \quad($ here $\gamma \geq 0)$;

(v) $f(x)=\alpha x^{2}+A(x)+\delta$

for all $x \in(0, \infty)$.

With the aid of this result we are able to prove a more general version of Theorem 2. Nevertheless, I believe that Theorem 2 with its more or less direct proof remains to be of interest for its own in the light of the fact that the proof of (JMP)-theorem presented in [4] is pretty long and sophisticated. Let me express at this place my sincere thanks to Professor Gy u la Maks a for drawing my attention to the paper [4] (see also [5]).

Theorem 3. Let $f, \varphi:(0, \infty) \longrightarrow \mathbb{R}$ be two strictly monotonic functions and let $\varphi$ be continuous. Then the pair $(f, \varphi)$ yields a solution to the functional equation

$$
f(x+y)=f(x)+f(y)+2 f\left(\varphi^{-1}\left(\frac{\varphi(x)+\varphi(y)}{2}\right)\right), \quad x, y \in(0, \infty),
$$

if and only if there exist nonzero real constants $c$ and $a$, and a real constant $b$ such that

$$
f(x)=c \cdot x^{2} \quad \text { and } \quad \varphi(x)=a \log x+b, \quad x \in(0, \infty) .
$$

Proof. Obviously, the function $h:=\frac{1}{2} \varphi$ is strictly monotonic. Since $\varphi$ is continuous, the domain $\varphi((0, \infty))$ of the inverse function $\varphi^{-1}$, being an interval, is convex which implies that the set

$$
D:=\{h(x)+h(y): x, y \in(0, \infty)\}
$$

coincides with the image $\varphi((0, \infty))$ and the function $g:=2 f \circ \varphi^{-1}$ yields a strictly monotonic map on $D$. Therefore we may apply (JMP)-theorem for a solution $f$ of equation $\left(\mathrm{E}_{\varphi}\right)$. Observe that equation $\left(\mathrm{E}_{\varphi}\right)$ on setting $y=x$ gives the relationship

$$
f(2 x)=4 f(x), \quad x \in(0, \infty) .
$$

On the other hand, each of possible solutions (i)-(v) is of the form $u+A$ where $u$ is a differentiable function and $A$ is additive. Therefore

$u(2 x)+2 A(x)=4 u(x)+4 A(x) \quad$ whence $\quad A(x)=\frac{1}{2} u(2 x)-2 u(x), \quad x \in(0, \infty)$. 
In particular, $A$ is continuous and hence linear: $A(x)=\lambda x, x \in(0, \infty)$. Consequently, in each of the cases considered $f$ is differentiable. The derivative $f^{\prime}$ satisfies the equality

In case (i) we get

$$
f^{\prime}(2 x)=2 f^{\prime}(x), \quad x \in(0, \infty)
$$

$$
f^{\prime}(x)=\alpha \beta \tanh (\beta x+\gamma)+\text { const }, \quad x \in(0, \infty),
$$

which fails to satisfy (1) because $\alpha \beta \neq 0$.

Case (ii) is eliminated in a similar way. In case (iii), we have

$$
f^{\prime}(x)=\alpha \beta e^{\beta x}+\lambda, \quad x \in(0, \infty),
$$

which also fails to satisfy 1. Finally, in case (iv) we have

$$
f^{\prime}(x)=\frac{\alpha}{x+\gamma}+\lambda, \quad x \in(0, \infty)
$$

and (11) is violated again. Therefore case (v) remains the only possible one; in this case one has $0=f^{\prime}(2 x)-2 f^{\prime}(x)=4 \alpha x+\lambda-4 \alpha x-4 \lambda$ which implies $\lambda=0$ and $f(x)=\alpha x^{2}, x \in(0, \infty)$. This finishes the proof because $\left(\mathrm{E}_{\varphi}\right)$ now gives the equality

$$
\varphi^{-1}\left(\frac{\varphi(x)+\varphi(y)}{2}\right)=\sqrt{x y}, x, y \in(0, \infty),
$$

and it suffices to apply Remark 1 to get the representation $\varphi(x)=a \log x+b$, $x \in(0, \infty)$.

\section{Concluding remarks}

As it has already been said, the strict monotonicity of both $f$ and $\varphi$ is required by the nature of the problem. For the price of more sophisticated proof (but still avoiding any appeal to (JMP)-theorem), instead of the smoothness of the functions in question assumed in Theorem 2, it would suffice to assume that the composition $f \circ \varphi^{-1}$ is a $C^{1}$-function. On the other hand, it is remarkable indeed that no regularity assumptions whatsoever are imposed on the unknown function $f$ in (JMP)-theorem. Unfortunately, we could not have the benefit of that because in our application of (JMP)-theorem we had to assume the strict monotonicity of the composition $f \circ \varphi^{-1}$.

Plainly, there exist reasonable means $M$ that are not quasi-arithmetic like, for instance, the logarithmic one. If that is the case, the question of solving the corresponding functional equation

$$
f(x+y)=f(x)+f(y)+2 f(M(x, y)), \quad x, y \in(0, \infty),
$$

remains open. 


\section{ABSTRACT PYTHAGOREAN THEOREM \& CORRESPONDING FUNCTIONAL EQUATIONS}

It is worth emphasizing that in some cases to get the desired assertion, the only assumption upon the height function $\Phi$ was just the reflexivity (cf. Theorem 1). It indicates that the requirement for $f$ to have the right-hand side derivative at zero is pretty strong indeed to force $f$ to be quadratic. What about possible alternate assumptions not involving boundary points of the domain?

Finally, the functional equation (E) seems to be interesting enough to be examined in detachment of its geometric background, e.g., for vector valued functions on abstract structures like groups or even semigroups. Till now that kind of studies remains untouched.

\section{REFERENCES}

[1] BERROnE, L. R.: The associativity of the Pythagorean law, Amer. Math. Monthly 116 (2009), 936-939.

[2] DUBIKAJTIS, L.: The axioms of S-geometry (oral communication; presented also at the symposium on geometry (Rome, July 1982)).

[3] GER, R.: A consistency equation for three geometries, Aequationes Math. 29 (1985), 50-55.

[4] JÁRAI, A.-MAKSA, GY.-PÁLES, ZS.: On Cauchy-differences that are also quasi sums Publ. Math. Debrecen 65 (2004), 381-398.

[5] MAKSA, GY.: Oral communication, The 10th Debrecen-Katowice Seminar on Functional Equations and Inequalities, 2010, Zamárdi, Hungary. 